\title{
Optimal Asset Allocation: A Worst Scenario Expectation Approach
}

\author{
Fei Lung Yuen ${ }^{* \dagger}$ and Hailiang Yang ${ }^{\ddagger}$
}

\begin{abstract}
Mean-variance criterion has long been the main stream approach in the optimal portfolio theory. The investors try to make a balance between the risk and return on their portfolio. In this paper, the deviation of the asset return from the investor's expectation in the worst scenario is taken as the measure of risk for portfolio selection. One important advantage of this approach is that the investors can base on their own knowledge, information and preference on various risks, in addition to the asset's volatility, to adjust their exposure to various risks. It also pinpoints one main concern of the investors when they invest, the amount they lose in the worst situation.
\end{abstract}

Keywords Asset allocation, risk measure, information uncertainty, worst case scenario, incomplete market

AMS Classification 90B, $91 \mathrm{G}$

\footnotetext{
*Acknowledgments We would like to thanks the editor, the associate editor and three anonymous referees for their help and useful comments on the paper. Hailiang Yang would like to acknowledge the Research Grants Council of the Hong Kong Special Administrative Region, China (project No. HKU 706611P).

$\dagger$ Department of Actuarial Mathematics and Statistics, and the Maxwell Institute for Mathematical Sciences, Heriot-Watt University, Edinburgh EH14 4AS, United Kingdom. E-mail: F.Yuen@hw.ac.uk, telephone number: 0131-451-8158

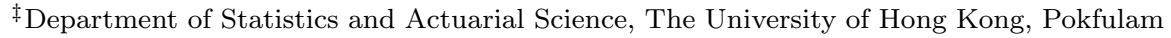
Road, Hong Kong. E-mail: hlyang@hku.hk, telephone number: 852-2857-8322
} 


\section{Introduction}

Asset allocation is one of the key research areas in finance. The classical approach of identifying the optimal portfolio for investors is to fix the risk and maximize the expected return or fix the required expected return and minimize the risk. Starting from the well known paper of Markowitz [1], variance

or standard derivation of the portfolio return is considered as the measure of risk and mean-variance approach is a popular way to find the optimal portfolio. Many papers have used this criterion to identify the optimal investment strategy, including Zhou and $\mathrm{Li}$ [3], $\mathrm{Li}$ and $\mathrm{Ng}$ [4], Chen et al. [5]. There are other approaches developed in the literature, including the utility function approach. Samuelson [6] applies the concept of utility function and considers the problem of optimal consumption and terminal wealth in asset allocation in a discrete model. Merton [2] extends the idea in Samuelson [6] to a continuous model. Merton [7] introduces an extra state variable in the market and identifies the resultant hedging demand in the optimal portfolio. Gennotte [8] studies the effects of estimation error in market parameters on asset allocation. Bawa [9], Harlow [10] and Sing and Ong [11] consider the downside risk of the portfolio. Li et al. [12] consider the dynamic portfolio selection problem using the safetyfirst approach. In this approach, the investors pay more attentions on the large loss which is modelled by a power function called lower partial moments on loss. Longstaff [13] maximizes the expected utility of the investors in an illiquid market. Bertsimas et al. [14] use shortfall as a risk measure and study the asset allocation problem. $\mathrm{Li}$ and $\mathrm{Ng}$ [4] extend the mean-variance approach to a multiperiod model and obtain the explicit form of the optimal investment strategy. Chen et al. [5] use this framework to deal with an asset-liability management problem.

Coherent risk measure suggested by Artzner et al. [15] becomes more and more popular in the area of risk management. Yang and Siu [16] and Elliott 
et al. [17] modify the probability measure to obtain a coherent measure of risk using generalized scenario expectation by considering the worst scenario and apply this in the area of risk management. The key concept of this coherent risk measure is to adjust the probability measure so that the worst market scenario is identified. Similar ideas are also used in Siu and Yang [18] and Gianin [19]. Unlike Value-at-Risk (VaR) which is a quantile of the loss, this measure is coherent and thus it is subadditive and consistent with the concept of diversification. In this paper, the loss based on this worst scenario expectation, instead of variance, is used as the measure of risk for a portfolio. In the area of risk management, the portfolio is known and so the worst scenario measure together with the expected loss can be identified directly. However, in asset allocation problem, the optimal strategy is the object that we want and it is unknown. It depends on the measure of risk which is determined by the worst scenario measure; at the same time, the worst scenario measure depends on the composition of the portfolio. Their interaction makes the optimal allocation problem more challenging.

When the return of the assets follows a normal distribution, it is natural that investors determine their optimal portfolio with information of risk and return of the assets, where risk is usually considered to be equivalent to the volatility of the assets. However, in general, what investors concern is loss and gain of money. They are happy to take an asset with high volatility if the high volatility comes from price appreciation. That is the reason why downside risk measures like semi-variance, shortfall, VaR and conditional tail expectation are introduced to measure the investment risk. Moreover, investors have their own idea on the return and risk, and thus the future price of assets, especially for the traders, analysts, economists and experienced investors. They identify the uncertainty based on the market information and select their optimal portfolio in a more conservative and more personal approach. Gennotte [8] gives a very inspiring study in this topic. Using the market data like volatility of the assets 
directly in asset allocation after personal adjustment makes more sense and is more practical. In this paper we bring the investors' knowledge into the model. The set of probability measures is now adjusted according to the investors' view on the assets, so using worst scenario expectation to represent the risk is more sound.

In this paper, we study the interaction between asset allocation and worst scenario expected loss using a constrained multiobjective optimization approach. We formulate the problem, simplify the required equations and solve the asset allocation problem in a simple market in Section 2. In Section 3, we consider a more general condition and apply our approach to an incomplete market. More economic interpretations of the results are shown in Section 4 and simple numerical examples are given in Section 5. The solution of the optimal asset allocation problem is found to be mathematically simple and consistent with the mean-variance and utility maximization approach.

\section{Problem Formulation}

We assume that there are $n+1$ assets and $n$ sources of Brownian motion risk in a complete market. Under the real probability measure $P$, which reflects the market information used by the investors, we denote $S_{i}(t)$ and $\mu_{i}(t)$ the price and the

expected rate of return for the $i^{t h}$ asset at time $t$. Let $\left(\Omega, \mathcal{F}, P,\left\{\mathcal{F}_{t}\right\}_{t \in[0, T]}\right)$ be a filtered probability space, $Z(t)=\left(Z_{1}(t), Z_{2}(t), \ldots, Z_{n}(t)\right)^{\prime}$ be the $n$-dimensinoal standard Brownian motion under this filtered probability space, and $Z^{\prime}$ denote the transpose of $Z$. Let $L_{\mathcal{F}}^{2}\left(0, T ; \mathbb{R}^{n}\right)$ be the set of square integrable, $\mathbb{R}^{n}$-valued stochastic process adapted to the filtration $\left\{\mathcal{F}_{t}\right\}_{t \in[0, T]}$. Let $S_{0}$ be the risk free asset and $r_{0}(t)$ be the risk free interest rate, we have

$$
d S_{0}(t)=r_{0}(t) S_{0}(t) d t
$$


We also have

$$
d S_{i}(t)=S_{i}(t)\left[\mu_{i}(t) d t+\sigma_{i}(t) d Z(t)\right]
$$

where $\sigma_{i}(t)$ is the $i^{t h}$ row of the matrix $\sigma(t)$ which summarizes the correlation between the rate of return of the risky assets and the Brownian motion risks. We denote $S(t):=\left(S_{1}(t), S_{2}(t), \ldots, S_{n}(t)\right)^{\prime}$ and $\mu(t):=\left(\mu_{1}(t), \mu_{2}(t), \ldots, \mu_{n}(t)\right)^{\prime}$. $\sigma(t)$ satisfies the following condition in this complete market,

$$
\|\sigma(t)\|>\epsilon \quad \forall t \in[0, T]
$$

for some $\epsilon>0$.

At time $t$, let $x(t)$ be the total wealth of the investor, $N_{i}(t)$ be the number of shares of asset $i$ held by the investor and $u_{i}(t)$ be the investor's position of asset $i$. Similarly, we denote $N(t):=\left(N_{1}(t), N_{2}(t), \ldots, N_{n}(t)\right)^{\prime}$ and $u(t):=$ $\left(u_{1}(t), u_{2}(t), \ldots, u_{n}(t)\right)^{\prime}$. Then,

$$
u_{i}(t)=N_{i}(t) S_{i}(t) \quad \forall i
$$

for all time $t$, if $\mathbf{1}_{n}$ is the n-dimensional column vector $(1,1, \ldots, 1)^{\prime}$ and define $\tilde{\mu}(t):=\mu(t)-r_{0}(t) \mathbf{1}_{n}$ as the risk premium rate of the risky assets, we have,

$$
\begin{aligned}
x(t) & =N_{0}(t) S_{0}(t)+N^{\prime}(t) S(t) \\
d x(t) & =N_{0}(t) d S_{0}(t)+N^{\prime}(t) d S(t) \\
& =N_{0}(t) r_{0}(t) S_{0}(t) d t+\sum_{i=1}^{n} N_{i}(t) S_{i}(t)\left[\mu_{i}(t) d t+\sigma_{i}(t) d Z(t)\right] \\
& =r_{0}(t) x(t) d t+\sum_{i=1}^{n} u_{i}(t)\left[\left(\mu_{i}(t)-r_{0}(t)\right) d t+\sigma_{i}(t) d Z(t)\right] \\
& =\left[r_{0}(t) x(t)+u^{\prime}(t) \tilde{\mu}(t)\right] d t+u^{\prime}(t) \sigma(t) d Z(t) .
\end{aligned}
$$

Investors want to have some profit, at least, expect some profit from the 
market when they are bearing the risk of the market. They think that with their knowledge and insight on the assets in the market, they are able to have a return higher than the risk free interest rate. However, the real return of the risky assets is stochastic. The investors have some ideas on the asset values in the market, but they are not sure whether the information they obtained from the market and their estimation of asset values are correct or not. They are conservative on their own evaluation on the assets' return in the market. Therefore, they consider a range of worst scenarios in order to identify the potential risk of the portfolio due to their misunderstanding on the economic environment. A range of conservative probability measures can be used where the size of the set of measures depends on the investors' degree of conservativeness. We assume that the set of measure is $\mathcal{Q}$. The relative entropy of $Q \in \mathcal{Q}$ and $P$ is

$$
K L(Q, P):=\int \frac{d Q}{d P} \ln \frac{d Q}{d P} d P \leq K \quad \forall Q \in \mathcal{Q} .
$$

The relative entropy measures the deviation of the real distribution from expectation. The parameter $K$ depends on the investors' confidence on the available information and their opinions on the market when they make an investment decision.

In most of the researches on asset allocation or risk, they use standard deviation and variance of the rate of return of the assets as the measure of risk. Recently, coherent measure of risk has caught much attention because it is consistent with various investment concepts and relatively robust. In this paper, we define the risk measure as the potential loss (deviation from expectation) of the portfolio due to incorrect judgement on the assets' return and it is equal to

$$
E_{P}(x(T))-\min _{Q \in \mathcal{Q}} E_{Q}(x(T)) .
$$

Similar definition of risk measure can be found in Yang and Siu (2001), Elliott 
et al. (2008). Suppose $a^{Q}(t):=\left(a_{1}^{Q}(t), a_{2}^{Q}(t), \ldots, a_{n}^{Q}(t)\right)^{\prime}$, let

$$
\frac{d Q}{d P}:=\exp \left(\int_{0}^{T}\left(a^{Q}\right)^{\prime}(t) d Z(t)-\frac{1}{2} \int_{0}^{T}\left(a^{Q}\right)^{\prime}(t) a^{Q}(t) d t\right) .
$$

Without loss of generality, we write $a^{Q}(t)$ as $a(t)$. We let $B_{0}(t):=S_{0}(T) / S_{0}(t)=$ $\exp \left(\int_{t}^{T} r_{0}(s) d s\right)$. By Girsanov theorem,

$$
\begin{aligned}
& E_{P}(x(T))=x(0) \exp \left(\int_{0}^{T} r_{0}(t) d t\right)+\int_{0}^{T} B_{0}(t) u^{\prime}(t) \tilde{\mu}(t) d t \\
& E_{Q}(x(T))=x(0) \exp \left(\int_{0}^{T} r_{0}(t) d t\right)+\int_{0}^{T} B_{0}(t) u^{\prime}(t)(\tilde{\mu}(t)+\sigma(t) a(t)) d t .(8)
\end{aligned}
$$

The risk measure can be simplified as

$$
-\min _{Q \in \mathcal{Q}} \int_{0}^{T} B_{0}(t) u^{\prime}(t) \sigma(t) a(t) d t
$$

Note that $K L(\mathcal{Q}, P):=\max _{Q \in \mathcal{Q}} K L(Q, P) \leq K$.

Using Lagrange principle, the constrained minimization problem above can be modified and we can find the optimal $Q$ (i.e. $a$ ) and the loss (risk measure) by considering a new problem. For some $\bar{\omega}>0$,

$$
\min _{Q \in \mathcal{Q}}\left[\int_{0}^{T} B_{0}(t) u^{\prime}(t) \sigma(t) a(t) d t+\bar{\omega}(K L(Q, P)-K)\right] .
$$

However, our goal is not just finding the worst scenario but using this worst scenario to determine the risk level and identify the optimal portfolio strategy. The investors try to maximize their wealth based on their information but at the same time control the exposure to risk as they are not totally confident on themselves due to the risk of the investment and the uncertainty of information. 
The problem now we are considering is

$$
\begin{aligned}
\text { Maximize } & E_{P}(x(T)) \\
\text { such that } & E_{P}(x(T))-\min _{Q \in \mathcal{Q}} E_{Q}(x(T)) \leq L, \\
\text { and } & K L(\mathcal{Q}, P) \leq K \\
\text { where } & x \text { satisfies (4) and } u \in L_{\mathcal{F}}^{2}\left(0, T ; \mathbb{R}^{n}\right) .
\end{aligned}
$$

Later, we implicitly assume that $x$ follows the dynamic of equation (4) and $u$ is integrable and adapted to the filtration. $L$ is the maximum amount of expected loss that the investor willing to endure for his portfolio and it reflects the degree of risk aversion of the investor. Here, $L$ is taken as an absolute amount but it can also depend on investor's wealth level, $x$. If the investor has a constant absolute risk aversion utility function, $L$ is a constant and independent of $x$; if the investor is constant relative risk aversion, we can take $L$ as the proportion of wealth. In this case, we let $l$ be the maximum expected proportional loss that the investor can tolerate in the worst scenario, and now $u$ can be viewed as the proportion of wealth in the risky assets. We can also choose $L$ proportional to $T$ if the investor expects a constant risk exposure at every instant and so risk tolerance level increases linearly with time. The risk characteristic of the investors controls the level of risk and the composition of their portfolio.

Our optimal asset allocation problem can be written as

$$
\begin{array}{cc}
\text { Maximize } & E_{P}(x(T)) \\
\text { such that } & \min _{Q \in \mathcal{Q}}\left[\int_{0}^{T} B_{0}(t) u^{\prime}(t) \sigma(t) a(t) d t+\bar{\omega}(K L(Q, P)-K)\right] \geq-L .
\end{array}
$$

We apply the theory of multiobjective optimization. As the set $\mathcal{Q}$ is convex and the loss constraint is linear with respect to the composition of portfolio, we can modify this constrained optimization problem into a single objective problem and the new objective function is the weighted average of the objectives of 
original problems. Similar applications can be found in Li and Ng (2000), Zhou and $\operatorname{Li}(2000)$. We let $\mathcal{U}=L_{\mathcal{F}}^{2}\left(0, T ; \mathbb{R}^{n}\right)$, the problem is further modified and becomes

$$
\max _{u \in \mathcal{U}}\left[E_{P}(x(T))+\lambda\left(\min _{Q \in \mathcal{Q}} \int_{0}^{T} B_{0}(t) u^{\prime}(t) \sigma(t) a(t) d t+\bar{\omega}(K L(Q, P)-K)+L\right)\right]
$$

where $\lambda>0$. With the expression of $\frac{d Q}{d P}$, we can obtain the $K L(Q, P)$ using the definition in equation (5),

$$
\begin{aligned}
K L(Q, P) & =\int \frac{d Q}{d P} \ln \frac{d Q}{d P} d P \\
& =\int\left(\int_{0}^{T} a^{\prime}(t)(d Z(t)-a(t) d t+a(t) d t)-\frac{1}{2} \int_{0}^{T} a^{\prime}(t) a(t) d t\right) d Q \\
& =\frac{1}{2} \int_{0}^{T} a^{\prime}(t) a(t) d t .
\end{aligned}
$$

Now, we focus on the optimal strategy and the corresponding worst scenario probability measure and ignore the constant and irrelevant terms. By using equation (7) and (8), the problem is simplified to

$$
\begin{aligned}
\max _{u \in \mathcal{U}}\left[\int_{0}^{T} B_{0}(t) u^{\prime}(t) \tilde{\mu}(t) d t+\lambda\left(\min _{Q \in \mathcal{Q}}\right.\right. & \int_{0}^{T} B_{0}(t) u^{\prime}(t) \sigma(t) a(t) d t \\
& \left.\left.+\bar{\omega}\left(\frac{1}{2} \int_{0}^{T} a^{\prime}(t) a(t) d t-K\right)+L\right)\right] .
\end{aligned}
$$

The optimal strategy and the corresponding worst scenario probability measure can be obtained by considering this minimax problem, with $\omega=\lambda \bar{\omega}$, we let

$$
\begin{aligned}
H(u, a):=\min _{Q \in \mathcal{Q}} \max _{u \in \mathcal{U}}\left[\int_{0}^{T} B_{0}(t) u^{\prime}(t) \tilde{\mu}(t) d t\right. & \\
& \left.+\lambda \int_{0}^{T} B_{0}(t) u^{\prime}(t) \sigma(t) a(t) d t+\frac{\omega}{2} \int_{0}^{T} a^{\prime}(t) a(t) d t\right], \\
h(t ; u, a):= & B_{0}(t) u^{\prime}(t) \tilde{\mu}(t)+\lambda B_{0}(t) u^{\prime}(t) \sigma(t) a(t)+\frac{\omega}{2} a^{\prime}(t) a(t) .
\end{aligned}
$$


For simplicity, we drop the time parameter $t$ and write $h(t ; u, a)$ as $h(u, a)$. We differentiate $h(u, a)$ with respect to $u$ and $a$. For all $t \in[0, T]$, we obtain

$$
\frac{\partial h(u, a)}{\partial u^{\prime}}=B_{0} \tilde{\mu}+\lambda B_{0} \sigma a, \quad \frac{\partial h(u, a)}{\partial a}=\lambda B_{0} u^{\prime} \sigma+\omega a^{\prime} .
$$

For the optimal strategy $u^{*}$ and worst scenario $a^{*}$, we have

$$
\tilde{\mu}+\lambda \sigma a^{*}=0, \quad \lambda B_{0} u^{* \prime} \sigma+\omega a^{* \prime}=0 .
$$

Together with the constraints of the original problem, we have

$$
\begin{aligned}
a^{*} & =-\frac{1}{\lambda} \sigma^{-1} \tilde{\mu} \\
\lambda & =\sqrt{\frac{\int_{0}^{T} \tilde{\mu}^{\prime} \sigma^{-1^{\prime}} \sigma^{-1} \tilde{\mu} d t}{2 K}}
\end{aligned}
$$

and so

$$
a^{*}=-\sqrt{\frac{2 K}{\int_{0}^{T} \tilde{\mu}^{\prime} \sigma^{-1^{\prime}} \sigma^{-1} \tilde{\mu} d t}} \sigma^{-1} \tilde{\mu} .
$$

We can also find $\omega$ and $u^{*}$,

$$
\begin{aligned}
\omega & =\frac{\lambda L}{2 K}=\frac{L}{2 K} \sqrt{\frac{\int_{0}^{T} \tilde{\mu}^{\prime} \sigma^{-1^{\prime} \sigma^{-1} \tilde{\mu} d t}}{2 K}}, \\
u^{*} & =\frac{-L}{2 K B_{0}} \sigma^{-1^{\prime}} a^{*} \\
& =L \frac{\sigma^{-1^{\prime}} \sigma^{-1} \tilde{\mu}}{2 K B_{0}}\left(\sqrt{\frac{\int_{0}^{T} \tilde{\mu}^{\prime} \sigma^{-1^{\prime} \sigma^{-1} \tilde{\mu} d t}}{2 K}}\right)^{-1} .
\end{aligned}
$$

After finding $a^{*}$ and $u^{*}$, we can obtain the expected absolute risk premium,

$$
\int_{0}^{T} B_{0} u^{* \prime} \tilde{\mu} d t=L \sqrt{\frac{\int_{0}^{T} \tilde{\mu}^{\prime} \sigma^{-1^{\prime}} \sigma^{-1} \tilde{\mu} d t}{2 K}} .
$$


The expected absolute risk premium enjoyed by the investor is proportional to their accepted amount of risk and for the uncertainty constraint $K$,

$$
\begin{aligned}
\frac{\partial\left(\int_{0}^{T} B_{0} u^{* \prime} \tilde{\mu} d t\right)}{\partial K} & =\frac{-L}{2 K} \sqrt{\frac{\int_{0}^{T} \tilde{\mu}^{\prime} \sigma^{-1^{\prime}} \sigma^{-1} \tilde{\mu} d t}{2 K}} \\
& =\frac{-1}{2 K} \int_{0}^{T} B_{0} u^{*^{\prime}} \tilde{\mu} d t
\end{aligned}
$$

To interpret the solutions obtained above, we can make use of the basic concept in pricing and risk management and the risk neutral probability measure $\widetilde{Q}$. As $\sigma$ summarizes the Brownian motion risks of the risky assets, $\sigma^{-1}$ is the collection of portfolios with exactly one unit of a particular Brownian motion risk. Therefore, $\sigma^{-1} \tilde{\mu}$ is the risk premium rate of the Brownian motion risk. Under risk neutral probability measure $\widetilde{Q}$,

$$
\begin{array}{rlr}
u^{\prime} \tilde{\mu}+u^{\prime} \sigma \tilde{a} & =0 \quad \forall u, t \\
\tilde{a} & =-\sigma^{-1} \tilde{\mu} \quad \text { so, } \\
K L(\widetilde{Q}, P) & =\frac{1}{2} \int_{0}^{T} \tilde{\mu}^{\prime} \sigma^{-1^{\prime}} \sigma^{-1} \tilde{\mu} d t .
\end{array}
$$

It is the term that appears in the expression of $\lambda, \omega, a^{*}$ and $u^{*}$. The price of wealth risk for the investor is

$$
\begin{aligned}
\frac{\text { Expected Return }}{\text { Potential Loss }} & =\frac{\lambda L}{L}=\lambda \\
& =\sqrt{\frac{\int_{0}^{T} \tilde{\mu}^{\prime} \sigma^{-1^{\prime}} \sigma^{-1} \tilde{\mu} d t}{2 K}} \\
& =\sqrt{\frac{K L(\widetilde{Q}, P)}{K}} .
\end{aligned}
$$

The price of wealth risk in the current model follows inverse square law of $K, K$ is the information uncertainty parameter. The more confidence the investors have on their estimation and the available information, the higher the expected profit 
they can enjoy. When $K=K L(\widetilde{Q}, P)$, one dollar risk faced by the investors provides them with expected profit of one dollar exactly.

We note that $K L(\widetilde{Q}, P)=\frac{1}{2} \int_{0}^{T} \tilde{a}^{\prime} \tilde{a} d t$. If $K<K L(\widetilde{Q}, P)$, the risk neutral probability measure is not in $\mathcal{Q}$ and the return of the risky asset is higher than the risk free rate in the worst scenario (assuming that risk premium rate is positive). The expected gain from investment is higher than the expected loss due to investor's uncertainty even in the worst scenario. When $K>K L(\widetilde{Q}, P)$, the risk neutral probability measure is now in $\mathcal{Q}$, in this case, investors are more uncertain about their estimation on the return of the risky assets. They are more conservative and they are going to reduce the amount of risky assets in the portfolio. They still invest in the risky asset as they believe that, in average, the risky assets can bring them a higher return than risk free asset, and they try to maximize this extra gain up to a risk level that they cannot tolerate. However, the risk premium (measured by expected asset appreciation) they have is now lower than the risk of expected loss they bear. This shows an interesting relationship between the pricing measure and the optimal asset allocation.

We assume that $\sigma$ is constant over time, all the $n$ Brownian motion risks are equivalent and they have the same risk premium rate, denote it by $\bar{\mu}$. Let $\bar{Q}$ be the risk neutral probability measure for this risk premium structure, then the optimal portfolio and the optimal total investment in risky asset are

$$
\begin{aligned}
u^{*} & =L \frac{\sigma^{-1^{\prime} \sigma^{-1} \mathbf{1}_{n}}}{2 K B_{0}}\left(\sqrt{\frac{\mathbf{1}_{n}^{\prime} \sigma^{-1^{\prime} \sigma^{-1} \mathbf{1}_{n} T}}{2 K}}\right)^{-1}, \\
u^{* \prime} \mathbf{1}_{n} & =\frac{L}{B_{0} T} \sqrt{\frac{\mathbf{1}_{n}^{\prime} \sigma^{-1^{\prime} \sigma^{-1} \mathbf{1}_{n} T}}{2 K}} \\
& =\frac{L}{B_{0} T} \sqrt{\frac{K L(\bar{Q}, P)}{\bar{\mu}^{2} K}} .
\end{aligned}
$$

We can see that the demand of risky assets by different investors and hence the 
whole market depends on the loss that investors are willing to bear and their discreetness on investment. Therefore, when the risk appetite of the market increases or the investors are more confident about the market information, the demand and thus the price of the risky assets increase. The expected risk premium at expiration earned at each time point is a constant and equal to

$$
u^{* \prime}\left(\bar{\mu} \mathbf{1}_{n}\right) B_{0}=\frac{L}{T} \sqrt{\frac{K L(\bar{Q}, P)}{K}} .
$$

\section{Different Understandings on Risks and Incom- plete Markets}

People can have different knowledge on different risks in the market. For example, an FX trader is more confident on predicting the interest rate trend than the oil price. For common investors, the risks of different sources with the same level of volatility are equivalent as they do not have any specific knowledge or confidence when facing any particular risk. For them, risk is volatility. However, for insiders or those experts in some areas of investment, finance, economy, properties and etc, they know more about some assets in the market, and they have their own ideas on investments. Even some assets seem to be highly volatile, they believe that the error of their estimation on the value of the assets and the real risk (not referring to volatility but losing money) is small. If these investors have different degrees of uncertain on the risks, the Brownian motion risks are not equivalent for them. They can use their knowledge and level of confidence on the estimations of the risk in the worst scenario (probability measure) in order to find their optimal strategy.

In this section we also discuss the market incompleteness. If the number of assets is greater than the number of the sources of risks, in a market with no arbitrage, there are some redundant assets and the portfolio construction can 
be done by removing all the redundant assets and we know that the optimal portfolio is no longer unique. If the number of assets is smaller than the number of risks, the market is incomplete and we have to do a little more in order to obtain the optimal portfolio.

Let $w(t):=\left(w_{1}(t), w_{2}(t), \ldots, w_{n}(t)\right)^{\prime}$ be the adjustments of the investor's relative confidence on the estimation of risks, and $\operatorname{diag}(w)$ be the diagonal matrix with vector $w$ as its principle diagonal. We suppose that $w>0$ and the new problem becomes

$$
\begin{array}{cc}
\text { Maximize } & E_{P}(x(T)) \\
\text { such that } & E_{P}(x(T))-\min _{Q \in \mathcal{Q}} E_{Q}(x(T)) \leq L, \\
\text { and } & \int_{0}^{T} a^{\prime}(t) \operatorname{diag}(w(t)) a(t) d t \leq K .
\end{array}
$$

Similar to the calculation in the previous section, it can be simplified as follows,

$$
\begin{array}{rc}
\text { Maximize } & \int_{0}^{T} B_{0}(t) u^{\prime}(t) \tilde{\mu}(t) d t \\
\text { such that } & \min _{Q \in \mathcal{Q}} \int_{0}^{T} B_{0}(t) u^{\prime}(t) \sigma(t) a(t) d t \geq-L, \\
\text { and } & \int_{0}^{T} a^{\prime}(t) \operatorname{diag}(w(t)) a(t) d t \leq K,
\end{array}
$$

and the optimization problem becomes

$$
\begin{gathered}
\max _{u \in \mathcal{U}}\left[\int_{0}^{T} B_{0}(t) u^{\prime}(t) \tilde{\mu}(t) d t+\lambda\left(\min _{Q \in \mathcal{Q}} \int_{0}^{T} B_{0}(t) u^{\prime}(t) \sigma(t) a(t) d t+\right.\right. \\
\left.\left.\bar{\omega}\left(\int_{0}^{T} a^{\prime}(t) \operatorname{diag}(w(t)) a(t) d t-K\right)+L\right)\right]
\end{gathered}
$$

Since we want to extend our study to an incomplete market, we propose that there are $m$ risky assets and $n$ sources of risks, where $m \leq n$. Then, for all 
$t \in[0, T]$,

$$
\tilde{\mu}(t), u(t) \in \mathbb{R}^{m}, \quad \sigma(t) \in \mathbb{R}^{m \times n}, \quad w(t), a(t) \in \mathbb{R}^{n} .
$$

We assume

$$
\begin{gathered}
G(u, a):=\min _{Q \in \mathcal{Q}} \max _{u \in \mathcal{U}}\left[\int_{0}^{T} B_{0}(t) u^{\prime}(t) \tilde{\mu}(t) d t+\lambda \int_{0}^{T} B_{0}(t) u^{\prime}(t) \sigma(t) a(t) d t\right. \\
\left.+\omega \int_{0}^{T} a^{\prime}(t) \operatorname{diag}(w(t)) a(t) d t\right], \\
g(t ; u, a):=B_{0}(t) u^{\prime}(t) \tilde{\mu}(t)+\lambda B_{0}(t) u^{\prime}(t) \sigma(t) a(t)+\omega a^{\prime}(t) \operatorname{diag}(w(t)) a(t) .
\end{gathered}
$$

Again, we suppress the time parameter $t$ if there is no ambiguity, and differentiate $g(u, a)$ with respect to $u$ and $a$, for all $t \in[0, T]$, we obtain,

$$
\frac{\partial g(u, a)}{\partial u}=B_{0} \tilde{\mu}+\lambda B_{0} \sigma a, \quad \frac{\partial g(u, a)}{\partial a}=\lambda B_{0} u^{\prime} \sigma+2 \omega a^{\prime} \operatorname{diag}(w) .
$$

The optimal strategy $u^{*}$ and worst scenario $a^{*}$ satisfy, for all $t \in[0, T]$,

$$
\tilde{\mu}+\lambda \sigma a^{*}=0, \quad \lambda B_{0} u^{* \prime} \sigma+2 \omega a^{* \prime} \operatorname{diag}(w)=0 .
$$

Together with the constraint equations, when $m=n$, we can apply the previous result by replacing $a^{*}$ by $\operatorname{diag}(\sqrt{w}) a^{*}, \sigma$ by $\sigma \operatorname{diag}(\sqrt{w})^{-1}$ and $\omega$ by $2 \omega$, where 
$\sqrt{w}:=\left(\sqrt{w_{1}}, \sqrt{w_{2}}, \ldots, \sqrt{w_{n}}\right)^{\prime}$. We have

$$
\begin{aligned}
\lambda & =\sqrt{\frac{\int_{0}^{T} \tilde{\mu}^{\prime} \sigma^{-1^{\prime}} \operatorname{diag}(w) \sigma^{-1} \tilde{\mu} d t}{K}}, \\
a^{*} & =-\sigma^{-1} \tilde{\mu}\left(\sqrt{\frac{\int_{0}^{T} \tilde{\mu}^{\prime} \sigma^{-1^{\prime}} \operatorname{diag}(w) \sigma^{-1} \tilde{\mu} d t}{K}}\right)^{-1}, \\
\omega & =\frac{L}{2 K} \sqrt{\frac{\int_{0}^{T} \tilde{\mu}^{\prime} \sigma^{-1^{\prime}} \operatorname{diag}(w) \sigma^{-1} \tilde{\mu} d t}{K}}, \\
u^{*} & =L \frac{\sigma^{-1^{\prime}} \operatorname{diag}(w) \sigma^{-1} \tilde{\mu}}{K B_{0}}\left(\sqrt{\frac{\int_{0}^{T} \tilde{\mu}^{\prime} \sigma^{-1^{\prime}} \operatorname{diag}(w) \sigma^{-1} \tilde{\mu} d t}{K}}\right)^{-1} .
\end{aligned}
$$

However, if $m<n$, we cannot use inverse matrix to obtain the solution directly. As we have discussed above, we can focus on the market without redundant assets (if there is a redundant asset, we can substitute its replicating portfolio in optimal allocation and the resultant portfolio will still be optimal) and $\sigma$ satisfies

$$
\left\|\sigma(t) \sigma^{\prime}(t)\right\|>\epsilon \quad \forall t \in[0, T]
$$

for some $\epsilon>0$. Inspired by the results in the case of $m=n$, we have

$$
\begin{aligned}
\lambda & =\sqrt{\frac{\int_{0}^{T} \tilde{\mu}^{\prime}\left(\sigma \operatorname{diag}(w)^{-1} \sigma^{\prime}\right)^{-1} \tilde{\mu} d t}{K}}, \\
\omega & =\frac{L}{2 K} \sqrt{\frac{\int_{0}^{T} \tilde{\mu}^{\prime}\left(\sigma \operatorname{diag}(w)^{-1} \sigma^{\prime}\right)^{-1} \tilde{\mu} d t}{K}}
\end{aligned}
$$


and,

$$
\begin{aligned}
u^{*} & =L \frac{\left(\sigma \operatorname{diag}(w)^{-1} \sigma^{\prime}\right)^{-1} \tilde{\mu}}{K B_{0}}\left(\sqrt{\frac{\int_{0}^{T} \tilde{\mu}^{\prime}\left(\sigma \operatorname{diag}(w)^{-1} \sigma^{\prime}\right)^{-1} \tilde{\mu} d t}{K}}\right)^{-1} \\
a^{*} & =\frac{-\lambda}{2 \omega}(\operatorname{diag}(w))^{-1} \sigma^{\prime} u^{*} \\
& =-(\operatorname{diag}(w))^{-1} \sigma^{\prime}\left(\sigma \operatorname{diag}(w)^{-1} \sigma^{\prime}\right)^{-1} \tilde{\mu}\left(\sqrt{\frac{\int_{0}^{T} \tilde{\mu}^{\prime}\left(\sigma \operatorname{diag}(w)^{-1} \sigma^{\prime}\right)^{-1} \tilde{\mu} d t}{K}}\right)^{-1}
\end{aligned}
$$

It can be easily shown that expressions above satisfy the optimal equations and constraint equations, hence, they are the optimal portfolio and the corresponding worst scenario. We also have one important result, adjustment on the relative entropy can be transformed to adjustment on volatilities for the corresponding risks in asset allocation problem. Hence, the investors' confidence on the assets' return can be summarized using adjusted volatilities.

\section{Efficient Frontier}

To illustrate the relationship between our approach and the mean-variance approach, we study the efficient frontier in our model. Now, rather than taking the $0^{\text {th }}$ asset as the risk free asset, we assume that it is also a risky asset and its price process satisfies

$$
d S_{0}(t)=S_{0}(t)\left[\mu_{0}(t) d t+\sigma_{0}(t) d Z(t)\right]
$$

Similar to the setting in the previous section, we consider a market with

$n$ Brownian motions and $m+1$ assets. We let $\hat{\mu}(t):=\mu(t)-\mu_{0}(t) \mathbf{1}_{n}$ and $\hat{\sigma}_{i}(t):=\sigma_{i}(t)-\sigma_{0}(t)$. We assume $n \geq m,\left\|\hat{\sigma}(t) \hat{\sigma}^{\prime}(t)\right\|>\epsilon$ for all $t \in[0, T]$ and 
some $\epsilon>0$. The wealth process becomes

$$
\begin{aligned}
d x(t)= & \left(x(t)-u^{\prime}(t) \mathbf{1}_{m}\right)\left(\mu_{0}(t) d t+\sigma_{0}(t) d Z(t)\right) \\
& \quad+\sum_{i=1}^{n} u_{i}(t)\left(\mu_{i}(t) d t+\sigma_{i}(t)\right) d Z(t) \\
= & \left(x(t) \mu_{0}(t)+u^{\prime}(t) \hat{\mu}(t)\right) d t+\left(x(t) \sigma_{0}(t)+u^{\prime}(t) \hat{\sigma}(t)\right) d Z(t) .
\end{aligned}
$$

We try to obtain the optimal portfolio by studying the asset allocation problem, let $B(t):=\exp \left(\int_{t}^{T} \mu_{0}(s) d s\right)$. The problem becomes

$$
\begin{aligned}
\text { Maximize } & \int_{0}^{T} B(t) u^{\prime}(t) \hat{\mu}(t) d t \\
\text { such that } & \min _{Q \in \mathcal{Q}} \int_{0}^{T} B(t)\left(u^{\prime}(t) \hat{\sigma}(t) a(t)+x(t) \sigma_{0}(t) a(t)\right) d t \geq-L, \\
\text { and } & \int_{0}^{T} a^{\prime}(t) \operatorname{diag}(w(t)) a(t) d t \leq K .
\end{aligned}
$$

We assume that the investors have constant relative risk aversion and $L$ is proportional to the wealth of the investors. Then, we let $l$ be the maximum expected proportional loss that the investor can tolerate in the worst scenario. The problem is changed to

$$
\begin{aligned}
\text { Maximize } & \int_{0}^{T} B(t) u^{\prime}(t) \hat{\mu}(t) d t \\
\text { such that } & \min _{Q \in \mathcal{Q}} \int_{0}^{T} B(t)\left(u^{\prime}(t) \hat{\sigma}(t) a(t)+\sigma_{0}(t) a(t)\right) d t \geq-l, \\
\text { and } & \int_{0}^{T} a^{\prime}(t) \operatorname{diag}(w(t)) a(t) d t \leq K,
\end{aligned}
$$

and now $u$ is understood as the proportion, but not the amount, of wealth the 
investors put in the $m$ assets. We assume

$$
\begin{gathered}
F(u, a):=\min _{Q \in \mathcal{Q}} \max _{u \in \mathcal{U}}\left[\int_{0}^{T} B(t) u^{\prime}(t) \hat{\mu}(t) d t+\lambda \int_{0}^{T} B(t)\left(u^{\prime}(t) \hat{\sigma}(t)\right.\right. \\
\left.\left.+\sigma_{0}(t)\right) a(t) d t+\omega \int_{0}^{T} a^{\prime}(t) \operatorname{diag}(w(t)) a(t) d t\right], \\
f(t ; u, a):=B(t) u^{\prime}(t) \hat{\mu}(t)+\lambda B(t)\left(u^{\prime}(t) \hat{\sigma}(t)+\sigma_{0}(t)\right) a(t) \\
+w a^{\prime}(t) \operatorname{diag}(w(t)) a(t) .
\end{gathered}
$$

Again, we omit the time parameter $t$ and we have

$$
\frac{\partial f(u, a)}{\partial u}=B \hat{\mu}+\lambda B \hat{\sigma} a, \quad \frac{\partial f(u, a)}{\partial a}=\lambda B\left(u^{\prime} \hat{\sigma}+\sigma_{0}\right)+2 \omega a^{\prime} \operatorname{diag}(w) .
$$

Solving the equations, we have,

$$
\begin{aligned}
\lambda & =\sqrt{\frac{\int_{0}^{T} \hat{\mu}^{\prime}\left(\hat{\sigma} \operatorname{diag}(w)^{-1} \hat{\sigma}^{\prime}\right)^{-1} \hat{\mu} d t}{K}}, \\
\omega & =\frac{l}{2 K} \sqrt{\frac{\int_{0}^{T} \hat{\mu}^{\prime}\left(\hat{\sigma} \operatorname{diag}(w)^{-1} \hat{\sigma}^{\prime}\right)^{-1} \hat{\mu} d t}{K}}, \\
a^{*} & =\frac{-(\operatorname{diag}(w))^{-1} \hat{\sigma}^{\prime}\left(\hat{\sigma} \operatorname{diag}(w)^{-1} \hat{\sigma}^{\prime}\right)^{-1} \hat{\mu}}{\lambda}, \\
u^{*} & =\left(\hat{\sigma} \operatorname{diag}(w)^{-1} \hat{\sigma}^{\prime}\right)^{-1}\left(\frac{l \hat{\mu}}{\lambda K B}-\hat{\sigma} \operatorname{diag}(w)^{-1} \sigma_{0}^{\prime}\right) .
\end{aligned}
$$

To study the relationship between expected value and variance of the return rate, we consider $w=I_{n}$. The expected rate of return $R$ of the optimal portfolio is

$$
u^{* \prime} \hat{\mu}+\mu_{0}=\frac{l}{\lambda K B} \hat{\mu}^{\prime}\left(\hat{\sigma} \hat{\sigma}^{\prime}\right)^{-1} \hat{\mu}-\sigma_{0} \hat{\sigma}^{\prime}\left(\hat{\sigma} \hat{\sigma}^{\prime}\right)^{-1} \hat{\mu}+\mu_{0}
$$

and the variance $V$ of the rate of return is

$$
\begin{aligned}
& \left(\sigma_{0}+u^{\prime} \hat{\sigma}\right)\left(\sigma_{0}+u^{\prime} \hat{\sigma}\right)^{\prime} \\
= & \sigma_{0} \sigma_{0}^{\prime}-\sigma_{0} \hat{\sigma}^{\prime}\left(\hat{\sigma} \hat{\sigma}^{\prime}\right)^{-1} \hat{\sigma} \sigma_{0}{ }^{\prime}+\left(\frac{l}{\lambda K B}\right)^{2} \hat{\mu}^{\prime}\left(\hat{\sigma} \hat{\sigma}^{\prime}\right)^{-1} \hat{\mu} .
\end{aligned}
$$


Therefore, the minimum variance frontier is

$$
V=\sigma_{0} \sigma_{0}^{\prime}-\sigma_{0} \hat{\sigma}^{\prime}\left(\hat{\sigma} \hat{\sigma}^{\prime}\right)^{-1} \hat{\sigma} \sigma_{0}{ }^{\prime}+\frac{\left(R-\left(\mu_{0}-\sigma_{0} \hat{\sigma}^{\prime}\left(\hat{\sigma} \hat{\sigma}^{\prime}\right)^{-1} \hat{\mu}\right)\right)^{2}}{\hat{\mu}^{\prime}\left(\hat{\sigma} \hat{\sigma}^{\prime}\right)^{-1} \hat{\mu}}
$$

and the upper part of the minimum variance frontier is the efficient frontier. Obviously, when $\sigma_{0}=0$ and $\mu_{0}=r_{0}$, the efficient frontier is equivalent to the Capital Market Line.

When $n=m$, there are $m+1$ assets in the market while there are only $n$ sources of risks. Asset 0 is redundant and can be replicated by a portfolio formed by the $m$ risky assets. The minimum variance frontier becomes

$$
V=\frac{\left(R-\left(\mu_{0}-\sigma_{0} \hat{\sigma}^{-1} \hat{\mu}\right)\right)^{2}}{\hat{\mu}^{\prime}\left(\hat{\sigma} \hat{\sigma}^{\prime}\right)^{-1} \hat{\mu}} .
$$

The market is completed by the risky assets and the price of return rate risk

is equal to $\sqrt{\hat{\mu}^{\prime}\left(\hat{\sigma} \hat{\sigma}^{\prime}\right)^{-1} \hat{\mu}}$. The risk free return of the market emerges and it is equal to $\mu_{0}-\sigma_{0} \hat{\sigma}^{-1} \hat{\mu}$.

When $n>m$, even in the case of $n=m+1$, we are not necessarily able to construct a risk free portfolio with non zero investment. The minimum variance portfolio is at the point where

$$
V=\sigma_{0} \sigma_{0}^{\prime}-\sigma_{0} \hat{\sigma}^{\prime}\left(\hat{\sigma} \hat{\sigma}^{\prime}\right)^{-1} \hat{\sigma} \sigma_{0}{ }^{\prime}, \quad R=\mu_{0}-\sigma_{0} \hat{\sigma}^{\prime}\left(\hat{\sigma} \hat{\sigma}^{\prime}\right)^{-1} \hat{\mu}
$$

\section{$5 \quad$ Numerical Examples and Illustrations}

We consider two simple numerical examples here to illustrate the idea of this model. We first assume that it is a complete market with three risky assets and three sources of Brownian motion risk. The investor has the following risk 
preference, information and ideas on the three assets,

$\sigma=\left(\begin{array}{ccc}0.1 & 0.15 & 0.1 \\ 0.2 & 0.05 & 0.2 \\ 0.25 & 0.1 & 0.1\end{array}\right) \quad \tilde{\mu}=\left(\begin{array}{c}10 \% \\ 13 \% \\ 13 \%\end{array}\right) \quad l=0.5 \quad K=6 \quad r_{0}=0.05$

in an one-year period. We now try to find the distribution of the investor's wealth in various assets.

Using the parameters given in equation above, we have

$$
\sigma^{-1}=\left(\begin{array}{rrr}
-4.0000 & -1.3333 & 6.6667 \\
8.0000 & -4.0000 & 0.0000 \\
2.0000 & 7.3333 & -6.6667
\end{array}\right) \quad \sigma^{-1} \tilde{\mu}=\left(\begin{array}{l}
29.33 \% \\
28.00 \% \\
28.67 \%
\end{array}\right) \quad \lambda=0.1434
$$

With $\lambda$, we can obtain the optimal investment strategy and the corresponding worst scenario measure

$$
u^{*}(0)=\left(\begin{array}{c}
0.4535 \\
0.1634 \\
0.0123
\end{array}\right) \quad a^{*}=\left(\begin{array}{c}
-2.0461 \\
-1.9531 \\
-1.9996
\end{array}\right) \quad B_{0} u^{* \prime} \tilde{\mu}=7.17 \%
$$

The result shows that the investor places $45.35 \%, 16.34 \%$ and $1.23 \%$ of his wealth on three risky assets correspondingly at time 0 . The remaining wealth will be put in risk free asset. He can obtain an extra $7.17 \%$ expected rate of return from the risky assets at time 1 in addition to the risk free rate. We can see that the risk premiums of different sources of risk are similar to each other, $a^{*}$ is negative and proportional to the risk premium. The worst situation occurs when the asset with the higher expected return gives a poorer result because a profit-seeking investors should invest more on this asset.

If one more Brownian motion risk is introduced into the market, the market is no longer complete and we try to use the approach in previous section to find 
the optimal portfolio. For a one-year investment plan, we assume that the new market condition is

$$
\sigma=\left(\begin{array}{cccc}
0.1 & 0.15 & 0.1 & 0.1 \\
0.2 & 0.05 & 0.2 & 0.1 \\
0.25 & 0.1 & 0.1 & 0.1
\end{array}\right) \quad \tilde{\mu}=\left(\begin{array}{c}
10 \% \\
13 \% \\
13 \%
\end{array}\right) \quad l=0.5 \quad K=4
$$

Further, suppose $w=(0.3,0.3,0.3,0.1)^{\prime}$, we obtain

$$
\lambda=0.1087 \quad u^{*}(0)=\left(\begin{array}{l}
0.1277 \\
0.1711 \\
0.1285
\end{array}\right) \quad a^{*}=\left(\begin{array}{c}
-2.2181 \\
-1.1372 \\
-1.6777 \\
-3.5940
\end{array}\right) \quad B_{0} u^{* \prime} \tilde{\mu}=5.44 \%
$$

From the selection of $w$, we know that the investor in this example is more uncertain about the information of the market data comparing to that in the previous example, especially for the new Brownian risk. However, we cannot compare the discreetness of the investors in these two examples because they are of two different markets and the definitions of $K$ are not the same. With the introducing of the new source of risk, the investor distributes his wealth more evenly on three risky assets, $12.77 \%, 17.11 \%, 12.85 \%$ of the investor's total wealth are invested in three risky assets, respectively, at time 0 . The remaining capital is invested in risk free asset. The worst scenario puts more emphasis on the risk that investor feels less confident about the information. The risk premium rate can be enjoyed by the investor over the year is $5.44 \%$.

We assume $\Sigma$ is the covariance matrix of the return rate of the assets, it is symmetric and given by

$$
\Sigma=\sigma \sigma^{\prime}, \quad \forall t \in[0, T]
$$

If the investors have no preference on the sources of risks, we recall that the 
optimal risky portfolio $u^{*}$ has the following expression,

$$
\begin{aligned}
u^{*} & =L \frac{\left(\sigma \sigma^{\prime}\right)^{-1} \tilde{\mu}}{K B_{0}}\left(\sqrt{\frac{\int_{0}^{T} \tilde{\mu}^{\prime}\left(\sigma \sigma^{\prime}\right)^{-1} \tilde{\mu} d t}{K}}\right)^{-1} \\
& =L \frac{\Sigma^{-1} \tilde{\mu}}{\lambda K B_{0}} .
\end{aligned}
$$

The optimal asset allocation among the risky assets in this model is linear with respect to the product of the inverse of the covariance matrix of the assets and the risk premiums of the Brownian motion risks. It is consistent with the existing results on asset allocation problems obtained by Duffie [20] using maximum utility criterion and Zhou and Li [3] using mean-variance approach. Therefore, the ratios of the risky assets in the optimal portfolios under all these models are the same. The size of the risky portfolio is proportional to the amount of risk $L$ that the investor is willing to take. It parallels the idea of leverage.

In previous sections, we use the amount of expected gain and the expected worst scenario loss as the measure of return and risk, respectively. We now consider the relationship between the expected rate of return and the volatility of the optimal portfolio. Again, $u$ is now taken as the proportion of wealth for different risky assets. Then, the risk premium rate (real rate of return of the portfolio) is equal to

$$
\tilde{\mu}^{\prime} u^{*}=l \frac{\tilde{\mu}^{\prime}\left(\sigma \operatorname{diag}(w)^{-1} \sigma^{\prime}\right)^{-1} \tilde{\mu}}{K B_{0}}\left(\sqrt{\frac{\int_{0}^{T} \tilde{\mu}^{\prime}\left(\sigma \operatorname{diag}(w)^{-1} \sigma^{\prime}\right)^{-1} \tilde{\mu} d t}{K}}\right)^{-1} .
$$


The volatility of the portfolio is

$$
\begin{array}{r}
\sqrt{u^{* \prime} \sigma \sigma^{\prime} u^{*}}=\frac{l}{K B_{0}} \sqrt{\tilde{\mu}^{\prime}\left(\sigma \operatorname{diag}(w)^{-1} \sigma^{\prime}\right)^{-1} \sigma \sigma^{\prime}\left(\sigma \operatorname{diag}(w)^{-1} \sigma^{\prime}\right)^{-1} \tilde{\mu}} \\
\left(\sqrt{\frac{\int_{0}^{T} \tilde{\mu}^{\prime}\left(\sigma \operatorname{diag}(w)^{-1} \sigma^{\prime}\right)^{-1} \tilde{\mu} d t}{K}}\right)^{-1} .
\end{array}
$$

We can obtain the price of return rate risk of the investor from

$$
\frac{\tilde{\mu}^{\prime}\left(\sigma \operatorname{diag}(w)^{-1} \sigma^{\prime}\right)^{-1} \tilde{\mu}}{\sqrt{\tilde{\mu}^{\prime}\left(\sigma \operatorname{diag}(w)^{-1} \sigma^{\prime}\right)^{-1} \sigma \sigma^{\prime}\left(\sigma \operatorname{diag}(w)^{-1} \sigma^{\prime}\right)^{-1} \tilde{\mu}}} .
$$

The demand of the assets depends on the investors' confidence on the information. The low demand of the assets which are considered more uncertain by the investors results in a low asset price, but at the same time a higher expected return. For those agents who are more confident on these assets, they invest more in these assets (relative to others) and enjoy a higher expected return (more precisely, a higher return-risk ratio). Therefore, for long term investment, investors should try to pay attention to those assets which are considered to be more uncertain by the investors. The higher return-risk ratio might also attract more investors to analyze these assets. Competition among the investors can reduce the information uncertainty for those assets.

If all investors in the market have no preference on the sources of risks, $w=\mathbf{1}_{n}$ and $\operatorname{diag}(w)=I_{n}$, the market price of risk becomes $\sqrt{\tilde{\mu}^{\prime}\left(\sigma \sigma^{\prime}\right)^{-1} \tilde{\mu}}$ and it is the slope of the Capital Market Line in the model. The investors form their optimal portfolio using the risk free asset and the market portfolio according to their risk preference and it is the idea of Mutual Fund Theorem. However, if the investors are allowed to have different views and preferences on different risks, the price of risks for the investors are different, and investors select risky portfolios with different compositions. They do not necessarily invest in the market portfolio as it is no longer efficient for them from their point of view. 
For investors with different levels of confidence on various sources of risks, $w$ is introduced to reflect the degree of confidence on the estimation of risk. This model is typically useful for this kind of investors, especially when they want to find the optimal strategy in an incomplete market. The worst scenario helps prevent exposing to excessive risk while their portfolio can still benefit from their insight on the market. When the investors are uncertain on their understanding of some risks, the worst scenario expectation approach makes these risks relatively worse than others and protects the investors from model and information uncertainty.

\section{Conclusions}

In this paper, we have applied the concept of coherent risk measure and information uncertainty in asset allocation and used them as the criterion for obtaining the optimal portfolio. In the proposed worst scenario approach, instead of just using the volatility, expected rate of return of the assets and the risk preference of the agents, the knowledge and information of investors on the market are now brought into consideration in the process of the optimal investment strategy determination. The investors invest more in the assets that they are more confident on their future price and enjoy a higher expected return under a preferred risk level controlled by assets' volatility and information uncertainty. This approach is rational, simple and at the same time consistent with the current asset allocation models. From the results obtained, we have identified a special relationship between the pricing measure and the optimal asset allocation. We also find that the levels of confidence (or uncertainty) on different sources of risk can be understood as adjustments on volatilities in asset allocation problem and therefore the optimal strategy is mathematically simple and easy to obtain. In the last art of the paper, we have applied the risk measure approach in incomplete markets and the optimal investment strategy 
is identified.

\section{References}

1. Markowitz, H.: Portfolio Selection. Journal of Finance 7(1), 77-91 (1952).

2. Merton, R. C.: Lifetime Portfolio Selection under Uncertainty: the Continuous-Time Case. Review of Economics and Statistics 51(3), 247-257 (1969).

3. Zhou, X. Y. and Li, D.: Continuous-Time Mean Variance Portfolio Selection: A Stochastic LQ Framework. Applied Mathematics \& Optimization $42,19-33(2000)$.

4. Li, D. and Ng, W. L.: Optimal Dynamic Portfolio Selection: Multiperiod Mean-Variance Formation. Mathematical Finance 10(3), 387-406 (2000).

5. Chen, P., Yang, H. and Yin, G: Markowitz's Mean-variance Asset-liability Management with Regime Switching: A Continuous Model. Insurance: Mathematics and Economics 43, 456-465 (2008).

6. Samuelson, P. A.: Lifetime Portfolio Selection By Dynamic Stochastic Programming. Review of Economics and Statistics 51(3), 239-246 (1969).

7. Merton, R. C.: An Intertemporal Capital Asset Pricing Model. Econometrica 41(5), 867-887 (1973).

8. Gennotte, G.: Optimal Portfolio Choice Under Incomplete Information. The Journal of Finance 41(3), 733-746 (1986). 
9. Bawa, V. S.: Safety-First, Stochastic Dominance, and Optimal Portfolio Choice. The Journal of Financial and Quantitative Analysis 13(2), 255-271 (1978).

10. Harlow, W. V.: Asset Allocation in a Downside-Risk Framework. Financial Analysis Journal 47(5), 28-40 (1991).

11. Sing, T. F. and Ong, S. E.: Asset Allocation in a Downside Risk Framework. Journal of Real Estate Portfolio Management 6(3), 213-223 (2000).

12. Li, D., Chan, T. F. and Wan, W. L.: Safety-First Dynamic Portfolio Selection. Dynamics of Continuous, Discrete and Impulsive Systems 4, 585-600 (1998).

13. Longstaff, F. A.: Optimal Portfolio Choice and the Valuation of Illiquid Securities. Review of Financial Studies 14(2), 407-431 (2001).

14. Bertsimas, D., Lauprete, G. J. and Samarov, A.: Shortfall as a Risk Measure: Properties, Optimization and Applications. Journal of Economic Dynamics \& Control 28, 1353-1381 (2004).

15. Artzner, P., Delbaen, F., Eber, J. and Heath, D.: Coherent Measures of Risk. Mathematical Finance 9(3), 203-228 (1999).

16. Yang, H. and Siu, T. K.: Coherent Risk Measures for Derivatives under Black-Scholes Economy. International Journal of Theoretical and Applied Finance 4(5), 819-835 (2001).

17. Elliott, R. J., Siu, T. K. and Chan, L.: A PDE Approach for Risk Measures for Derivatives with Regime Switching. Annals of Finance 4, 55-74 (2008).

18. Siu, T. K. and Yang, H.: A PDE Approach to Risk Measures of Derivatives. Applied Mathematical Finance 7, 211-228 (2000). 
19. Gianin, E. R.: Risk Measures via g-expectations. Insurance: Mathematics and Economics 39, 19-34 (2006).

20. Duffie, D.: Dynamic Asset Pricing Theory. Princeton University Press, Princeton (2001). 